[1] M. H. B. Stowell, T. M. McPhillips, D. C. Rees, S. M. Soltis, E. Abresch, G. Feher, Science 1997, 276, 812.

[2] H. Michel, J. Deisenhofer, Biochemistry 1988, $27,1$.

[3] B. A. Diner, F. Rappaport, Annu. Rev. Plant Biol. 2002, 53, 551.

[4] P. Fromme, J. Kern, B. Loll, J. Biesiadka, W. Saenger, H. T. Witt, N. Krauss, A. Zouni, Philos. Trans. R. Soc. London, Ser. B 2002, 357, 1337.

[5] J. P. Allen, G. Feher, T. O. Yeates, H. Komiya, D. C. Rees, Proc. Natl. Acad. Sci. USA 1988, 85, 8487.

[6] O. El-Kabbani, C. H. Chang, D. Tiede, J. Norris, M. Schiffer, Biochemistry 1991, 30, 5361

[7] A. J. Chirino, E. J. Lous, M. Huber, J. P. Allen, C. C. Schenck, M. L. Paddock, G. Feher, D. C. Rees, Biochemistry 1994, 33, 4584.

[8] B. Arnoux, F. Reiss-Husson, Eur. Biophys. J. 1996, 24, 233.

[9] U. Ermler, G. Fritzsch, S. K. Buchanan, H. Michel, Structure 1994, 2, 925.

[10] E. Abresch, M. L. Paddock, M.H. B. Stowell, T. M. McPhillips, H. L. Axelrod, S. M. Soltis, D. C. Rees, M. Y. Okamura, G. Feher, Photosynth. Res. 1998, 55, 119.

[11] J. Deisenhofer, H. Michel, EMBO J. 1989, 8, 2149.

[12] J. Deisenhofer, O. Epp, I. Sinning, H. Michel, J. Mol. Biol. 1995, 246, 429.

[13] C. R. D. Lancaster, U. Ermler, H. Michel in Adv. Photosynth. 2: Anoxygenic Photosynthetic Bacteria, Vol. 2, Kluwer Academic, Dordrecht, The Netherlands, 1995.

[14] C. R. D. Lancaster, Biochim. Biophys. Acta 1998, 1365, 143.

[15] C. R. D. Lancaster, Biochem. Soc. Trans. 1999, 27, 591.

[16] D. Tiede, Biochemistry 1997, 35, 10763.

[17] O. A. Gopta, D. A. Bloch, D. A. Cherapanov, A. Y. Mulkidjanian, FEBS Lett 1997, 412, 490.

[18] J. Li, D. Gilroy, D. M. Tiede, M. R. Gunner, Biochemistry 1998, 37, 2818.

[19] E. G. Alexov, M. R. Gunner, Biochemistry 1999, 38, 8253.

[20] B. Rabenstein, G. M. Ullmann, E.-W. Knapp, Biochemistry 2000, 39, 10487.

[21] H. Ishikita, G. Morra, E.-W. Knapp, Biochemistry 2003, 42, 3882

[22] A. K. Grafton, R. A. Wheeler, J. Phys. Chem. B 1999, 103, 5380.

[23] S. E. Walden, R. A. Wheeler, J. Phys. Chem. B 2002, 106, 3001.

[24] J. Breton, C. Boullais, C. Mioskowski, P. Sebban, L. Baciou, E. Nabedryk, Biochemistry 2002, 41, 12921.

[25] Simulations of the Rhodopseudomonas viridis reaction center also imply that with $\mathrm{UQ}_{B}{ }^{\prime} \mathrm{S}$ head group oriented as in the distal X-ray structure it cannot migrate to the proximal site and the space between protein helices is too restricted to allow head group rotation: U. Zachariae, C. R. D. Lancaster, Biochim. Biophys. Acta 2001, 1505, 280.

[26] H. M. Berman, J. Westbrook, Z. Feng, G. Gilliland, T. N. Bhat, H. Weissig, I. N. Shindyalov, P. E. Bourne, Nucleic Acids Res. 2000, 28, 235.

[27] AMBER 6.0, D. A. Case, D. A. Pearlman, J. W. Caldwell, T. E. Cheatham, III. W. S. Ross, C. L. Simmerling, T. A. Darden, K. M. Merz, R. V. Stanton, A. L. Cheng, J. J. Vincent, M. Cowley, D. M. Ferguson, R. J. Radmer, G. L. Seibel, U.C. Singh, P.K. Weiner, P.A. Kollman, University of California: San Francisco, CA, 1997.

[28] D. A. Pearlman, J. W. Cladwell, W. S. Ross, T. E. Cheatham,Ill., S. DeBolt, D. Ferguson, G. Seibel, D. A. Case, P. A. Kollman, Comput. Phys. Commun. 1995, 91, 1.

[29] W. F. Van Gunsteren, H. J. C. Berendsen, Mol. Phys. 1977, 34, 1311.

[30] J. P. Ryckaert, G. Ciccotti, H. J. C. Berendsen, J. Comput. Phys. 1977, 23, 327.

[31] M. L. Paddock, P. Aedelroth, G. Feher, M. Y. Okamura, J.T. Beatty, Biochemistry 2002, 41, 14716.

[32] A. Mezzetti, E. Nabedryk, J. Breton, M. Y. Okamura, M. L. Paddock, G. Giacometti, W. Leibl, Biochim. Biophys. Acta 2002, 1553, 320

[33] W. D. Cornell, P. Cieplak, C.I. Bayly, I. R. Gould, K. M. Merz, Jr., D. M. Ferguson, D. C. Spellmeyer, T. Fox, J. W. Caldwell, P. A. Kollman, J. Am. Chem. Soc. 1995, 117, 5179.

[34] W. L. Jorgensen, J. Chandrasekhar, J. D. Madura, R. W. Impey, M. L. Klein, J. Chem. Phys. 1983, 79, 926.

[35] S. E. Boesch, R. A. Wheeler, J. Phys. Chem. A 1997, 101, 5799.

[36] R. A. Wheeler, J. Am. Chem. Soc. 1994, 116, 11048.

[37] S. E. Boesch, A. K. Grafton, R. A. Wheeler, J. Phys. Chem. 1996, 100, 10083.

[38] R. A. Wheeler, in Molecular bioenergetics: simulations of electron, proton, and energy transfer (Ed.: R. A. Wheeler), American Chemical Society, Washington DC, 2004, in press.

[39] WebLab ViewerPro, 4.0 Ed., Molecular Simulations, Inc, San Diego, CA, 2000

[40] C. Simmerling, R. Elber, J. Zhang in Modeling of Biomolecular Structure and Mechanisms (Ed.: A. Pullman), Kluwer, Norwell, MA, 1995, pp. 241.

Received: September 4, 2003 [Z961]

\section{Mechanical Properties of Microtubules Explored Using the Finite Elements Method}

\author{
Sandor Kasas, ${ }^{*[a, b]}$ András Kis, ${ }^{[c]}$ Beat Michel Riederer, ${ }^{[b]}$ \\ Lászlo Forró, ${ }^{[c]}$ Giovanni Dietler, ${ }^{[d]}$ and Stefan Catsicas ${ }^{[a]}$
}

Microtubules (MTs) are complex protein polymers composed of $\alpha$ and $\beta$ tubulin dimers which assemble into threads called protofilaments. Between eight and nineteen protofilaments may form a hollow tube of several micrometers long. ${ }^{[1-6]}$ The most common MTs, 13 protofilament MTs, have a diameter of $24 \mathrm{~nm}$ with a wall about $5 \mathrm{~nm}$ thick and a hollow central core about $15 \mathrm{~nm}$ in diameter. These dimensions are directly related to the number of protofilaments. MTs are one of the main components of the cytoskeleton and play an essential role in many fundamental physiological processes in the cell. They provide mechanical stability and maintain the cell's shape. Inside cells they act as railways along which motor proteins transport vesicles or organelles. During cell division they form the mitotic spindle, which is responsible for separating chromosomes that carry the genetic code. They can also form complex bundles (cilia and flagella) that can propel sperms and some eukaryotic cells (e.g., Euglena rostrifera). Microtubules assembled inside cells can be decorated with microtubule-associated proteins (MAPs) that can modify their spatial organization and dynamics. ${ }^{[7]}$

Mechanical properties of MTs largely determine their functions. Quantifying the way they resist mechanical deformation by determining their Young's and shear modulus can permit a better understanding of all the vital physiological mechanisms in which MTs are involved. However, measuring and understanding MTs mechanical properties is not a simple task. Two decades of measurements involving different techniques such as optical tweezers, ${ }^{[8]}$ hydrodynamic flow $^{[9]}$ atomic force microscope $(\mathrm{AFM}),{ }^{[10,11]}$ and persistence length observations, ${ }^{[12]}$ resulted in values of Young's modulus between $1 \mathrm{MPa}^{[10]}$ and $7 \mathrm{GPa}^{\left[{ }^{[8]}\right.}$ In all of these experiments, microtubules have been bent in some way and modeled as homogeneous, isotropic beams in order to calculate the Young's modulus from the experimental data.

[a] Dr. S. Kasas, Prof. S. Catsicas

Laboratoire de Neurobiologie Cellulaire

Faculté des Sciences de la Vie

Ecole Polytechnique Fédérale de Lausanne

1015 Lausanne (Switzerland)

E-mail: sandor.kasas@ipmc.unil.ch

[b] Dr. S. Kasas, Dr. B. M. Riederer

Institut de Biologie Cellulaire et de Morphologie, rue du Bugnon 9

Université de Lausanne, 1005 Lausanne (Switzerland)

[c] Dr. A. Kis, Prof. L. Forró

Institut de la Physique de la Matière Complexe

Ecole Polytechnique Fédérale de Lausanne

1015 Lausanne (Switzerland)

[d] Prof. G. Dietler

Institut de Physique de la Matière Condensée

Université de Lausanne, 1015 Lausanne (Switzerland) 
In the general case, the resistance of an object to bending is described by its flexural rigidity (bending stiffness). If MTs were homogeneous and isotropic, the bending stiffness would correspond with the Young's modulus $E_{\text {Young, }}$ and could be written as $E_{\text {Young }} l$, with / being the second moment of the tube's area. However, electron microscopic structural data clearly show that MTs are built of sparsely connected protofilaments, ${ }^{[13]}$ so this assumption is far from being true.

According to the most recent measurements on microtubules that had been immobilized on porous surfaces and deformed by an AFM tip, ${ }^{[11]}$ a lower limit of $100 \mathrm{MPa}$ can be set on the Young's modulus. The shear modulus, which describes the resistance of protofilaments to sliding with respect to each other, is $1.4 \mathrm{MPa}$. These results demonstrate that MTs are highly anisotropic from the mechanical point of view. Therefore, the flexural rigidity is no longer an adequate quantity for their description as it strongly varies as a function of length: short microtubules are flexible due to a low value of the shear modulus while longer tubes become more rigid-which is when the Young's modulus dominates the mechanical behavior. Measurements on longer microtubules would therefore provide better estimates of the Young's modulus, because neglecting the influence of shearing would introduce a smaller error. Experiments involving the longest microtubules $(24-68 \mu \mathrm{m})$ were performed by Mickey and Howard ${ }^{[14]}$ yielding a value of $2 \mathrm{GPa}$ for microtubules assembled from pure bovine-brain tubulin. Further credibility to this particular value is given by the fact that it lies close to the Young's modulus of similar proteins like actin, collagen, keratin, and isolated $\alpha$-helices - all of which haveg a Young's modulus of around $2 \mathrm{GPa} .{ }^{[15]}$ There is no general agreement on the influence of MAPs on the mechanical properties of microtubules. While some authors conclude that neither tau ${ }^{[14]}$ nor bovine brain MAPs have an appreciable effect, ${ }^{[16]}$ others find a fourfold increase of rigidity after the addition of MAPs. ${ }^{[17]}$

As a consequence of the neglect of the mechanical anisotropy of microtubules, all the previous experimental data that was analyzed using the assumption that microtubules are isotropic will have to be revisited and reinterpreted with more adequate models. At this stage, three types of theoretical MT models are available: analytical, molecular dynamics, and finite-elementbased models.

Analytical solutions for interpreting experimental data and simulating MT behavior are available only for a limited number of situations and at a price of dramatic simplifications. They can even cause considerable errors, as the problem of microtubule's mechanical anisotropy clearly demonstrates. Molecular dynamics (MD) would probably be the method of choice, but it is unfortunately not suitable for calculations on structures that can extend up to hundreds of micrometers. Additionally, MD simulations require a huge computational power which is not accessible to the majority of our community.

A more convenient way of modeling microtubules is by building a computer model and simulating its behavior under mechanical stress using the finite elements method (FEM). It enables the simulation and understanding of a richer variety of experimental situations than can be analytically solved. This method can also lead to building better analytical models by showing which assumptions can safely be made.

Contrarily to molecular dynamics, FEM analysis requires less computational power and runs on modern desktop computers. In addition, several user-friendly computer packages are commercially available, which gives the majority of our community the opportunity to model complex geometries and analyze their mechanical properties in a relatively short period of time.

Herein, we used FEM to validate assumptions of the analytical model, which has been used by Kis et al. ${ }^{[11]}$ We also used a similar MT model to explore the mechanical properties of microtubules that are missing an increasing amount of their tubulin components, corresponding to a situation that could occur when microtubules are exposed to oxidative stress. Finally, the FEM MT model was used to explore the mechanical properties of an unusual form of MT. Most of the microtubules are assembled from 13 protofilaments. ${ }^{[6]}$ However, many exceptions have been noted in different species and cell types. Various MT types having between eight and ninteen protofilaments were reported in the literature..$^{[1,2-6]}$ Different protofilament numbers are often found in different cell types-an indication that they might have distinct functions. For example, bovine-brain microtubules are almost exclusively composed of 13 protofilament MTs, ${ }^{[18]}$ yeast cells have mostly 12 protofilament MTs, while the nematode worm C. elegans has 11,13 , and 15 protofilament microtubules. ${ }^{[4]}$ Deletion of the 15 protofilament MT from C. elegans leads to the loss of sensitivity to touch, ${ }^{[4]}$ clearly demonstrating a specific biological role of this MT type.

Tubulin assembles to form MTs that differ by their protofilament and monomer helix start number. Lateral contacts are made between $\alpha-\alpha$ and $\beta-\beta$ subunits, except for a "seam" of $\alpha-\beta$ contacts. ${ }^{[19]}$ The microtubule lattice is believed to accommodate different configurations by skewing the protofilaments so that the continuity of the tubulin lattice is maintained. ${ }^{[6]}$ In a 13 protofilament MT (13:3) the lateral path of subunits closes exactly three subunits $(13: 3)$ above its starting position along the protofilament, giving rise to a three start helix organization in which the protofilaments remain parallel to the MT axis. On the other hand, in a 15 protofilament MT (15:3), the three start monomer helix (15:3) is slightly out of register compared to its ideal position in the 13:3 MT. To correct this mismatch, protofilaments become slightly skewed and "turn" helically around the MT axis. Electron-microscopy studies have confirmed that significantly different surface lattices such as (14:3) and (15:4) can have essentially the same inter-protofilament contacts. ${ }^{[20]}$ This extraordinary ability of the same object, the MT lattice to accommodate different structures by skewing is made easier by their mechanical anisotropy: weak interaction between the protofilaments makes their relative displacement by shearing easy.

Given that MTs can adopt different lattice structures and since their mechanical properties determine their function, a question arises about the advantages nature has in preferring one configuration over the other. Different forms of MT could have different stiffness or resistance to buckling and structural defects. Experiments which would address these questions are difficult to implement, because in addition to the intrinsic 
difficulty of performing the measurement itself, there is a necessity to isolate MTs with the right protofilament number. Because there is a large number of potentially interesting MT types, computer simulation could show which microtubules would be especially interesting for further experimental studies. We therefore applied FEM modeling to analyze the mechanical behavior under mechanical stress of the 15:3 MT, because the 15 protofilament microtubules have been shown to play a crucial role in the touch-sensitivity of $C$. elegans. ${ }^{[4]}$

The aim of this set of simulations was to validate assumptions of the analytical model which has been used by Kis et al. to determine the Young's and shear moduli of microtubules. ${ }^{[11]}$

In that work, MTs assembled from pure bovine brain tubulin (without MAPs) were deposited onto a surface of PMMA onto which slits have been cut using electron-beam lithography (Figure 1 and 2A). Suspended segments of the MT were

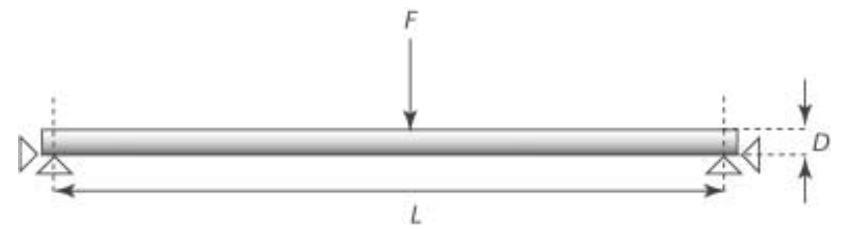

Figure 1. Schematic depiction of a microtubule in the suspended-beam configuration. A point load $\mathrm{F}$ is applied in the middle of the suspended segment of length $\mathrm{L}$ and diameter $\mathrm{D}$.
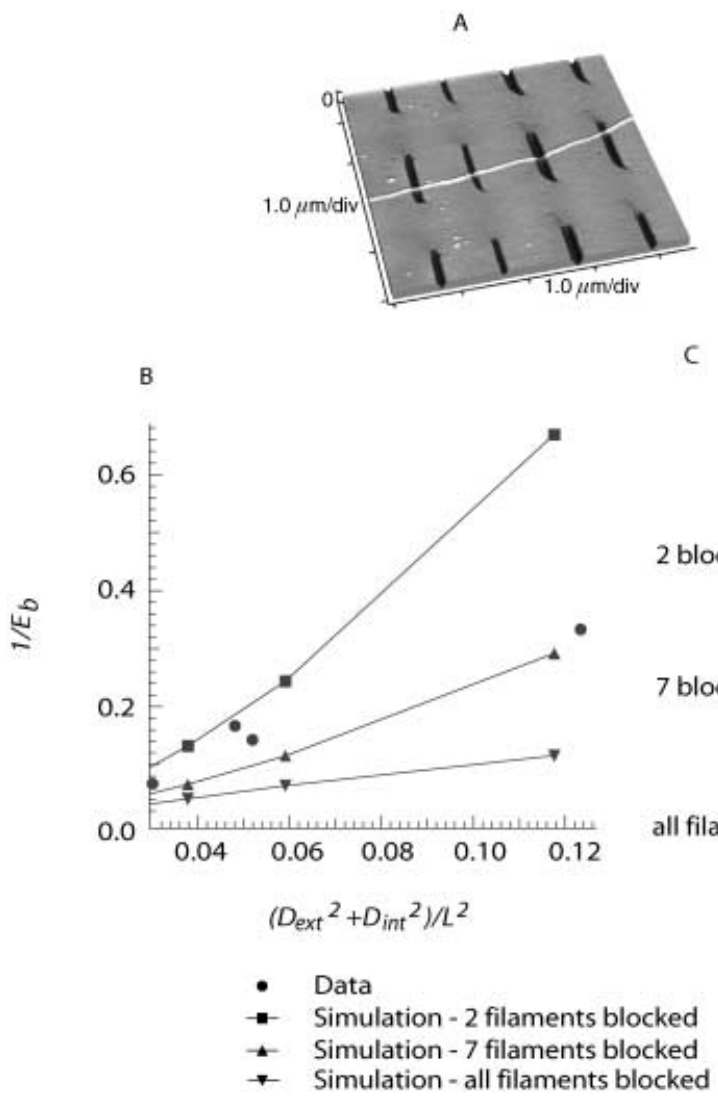

Figure 2. A) An AFM image of a microtubule bridging different sized holes in PMMA. B) Comparison of simulated with the experimental data ${ }^{[11]}$ for the boundary conditions schematically depicted in (C). deformed by the tip of an AFM and the amount of deformation permitted to deduce simultaneously the Young's and the shear modulus of MT from the variation of the flexural rigidity as a function of suspended length. If we model the microtubule as an anisotropic beam clamped at both ends, its deformation can, in general, be described as a sum of the deformation due to bending $\delta_{\mathrm{B}}$ and the deformation due to shearing $\delta_{\mathrm{S}}$ [Equation (1)]:[21]

$\delta=\delta_{\mathrm{B}}+\delta_{\mathrm{S}}=\frac{F L^{3}}{192 E_{\text {Young }} I}+f_{\mathrm{s}} \frac{F L}{4 G A}=\frac{F L^{3}}{192 E_{\text {bending }} I}$

where $F$ is the applied normal load, $L$ the suspended length, $E_{\text {Young }}$ the Young's modulus, $G$ the shear modulus, $I$ is the second moment of the cross-sectional area $A$, and $f_{s}$ a numerical factor that can range from $1-2$, depending on the geometry, and is equal to $10 / 9$ for a filled cylinder. The overall mechanical response can be described by the length-dependent bending modulus $E_{\text {bending }}$ [Equation (2)],

$\frac{1}{E_{\text {bending }}}=\frac{1}{E_{\text {Young }}}+\frac{10\left(D_{\text {ext }}+D_{\text {int }}\right)^{2}}{3 G L^{2}}$

which is the result of a single bending experiment. $D_{\text {int }}$ and $D_{\text {ext }}$ are respectively the tube's internal and external diameters. In order to extract the values of $E_{\text {Young }}$ and $G$, measurements have to be performed in which the suspended length $L$ varies .

In our simulation, a 13:3 MT was "suspended" above "holes" of different lengths. The solid surface on which the MT was lying was simulated by blocking the MT lower protofilaments. A schematic of the construction is represented in Figure 1.

Curves depicting MT deformation as a function of applied force all show linear behavior. Varying the hole size reproduced the essential feature of the AFM experiment: dependence of $E_{\text {bending }}$ on the suspended length (Figure $2 \mathrm{~b}$ ).

We also tested the assumption that microtubule behaves as a clamped beam by changing the number of protofilaments that are blocked by the substrate. Results for the variation of $E_{\text {bending }}$ as a function of length for two, seven, and thirteen blocked protofilaments are shown in Figure $2 \mathrm{~b}$. The main criterion for comparisons with the experiment is the overall shape of the curve, which describes the dependence of $E_{\text {bending }}$ on MT length. Deviations of this curve from a straight line in Figure $2 \mathrm{~b}$ show how much of the length dependence of the $E_{\text {bending }}$ value is an artifact that comes from the boundary conditions and how much from real, physical properties of MTs. The absolute numerical values depend on the calibration itself. As we didn't want our simulations to favor any of the possible extremes in the number of blocked filaments, we calibrated the shear modulus in our model by assuming that only the seven lower protofilaments were bound in the experiment. During calibration, the slope of the linear fit of the simulated points was adjusted to the slope of the experimental values. Because we were using a fixed, predetermined value of the Young's modulus, the actual points didn't overlap.

Results where only two protofilaments were blocked show a significant deviation from the experimental data and the 
behavior described by Equation (2) at small suspended lengths. In the case where all the protofilaments have been blocked, which corresponds to the clamped-beam model, the linear dependence of $1 / E_{\text {bending }}$ on $\left[\left(D_{\text {ext }}{ }^{2}+D_{\text {int }}{ }^{2}\right) L^{-1}\right]^{2}$ is completely reproduced, while in the case of seven blocked protofilaments there is still a small deviation at small lengths.

The most significant influence of varying the boundary conditions is the deviation of the mechanical behavior from Equation (2), while the exact numerical values and their eventual accordance with the experiment depend on model calibration. As the experimental data shows a linear behavior that is qualitatively the same as in the simulation with all protofilaments blocked, we conclude that the original assumption of a clamped beam is a valid approximation. The experimentally observed variation of the microtubule's $E_{\text {bending }}$ value is therefore physical, and not an artifact induced by the boundary conditions.

Most of the measurements of the MT mechanical properties assumed that these filamentous protein assemblies have a homogenous and isotropic structure. To estimate the extent of discrepancy in the mechanical behavior between the isotropic and the anisotropic cases, we simulated two different kinds of MTs. Isotropic 13:3 MTs were simulated by setting the same material constants for all the connections between single tubulin molecules, (2 GPa each) whereas anisotropic ones had different materials constants along and between their protofilaments ( $2 \mathrm{GPa}$ for the Young's modulus and 1.4MPa for the shear modulus). Both MTs were fixed at one of their ends (setting all degrees of freedom (DOF) to zero for the concerned nodes) and a known transversal force was applied to their remaining free end. The lengths of both MTs were set to $2 \mu \mathrm{m}$; a length-scale that is typical to most of the experiments reported in the literature. The same transversal force induced a $70 \%$ larger bending in the anisotropic than in the isotropic MT. Even when the end-deformations are adjusted to be equal, there is a significant difference in the overall shape of the deformed microtubule (Figure 3), which could drastically influence estimations of the microtubule's persistence length. ${ }^{[12]}$

In the simulation run to investigate the influence of the random suppression of tubulin molecules from an MT on its

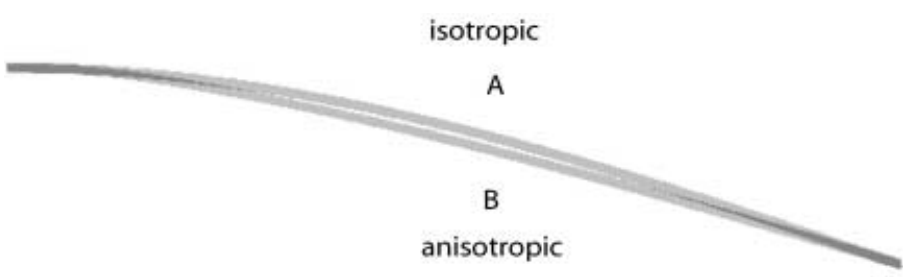

Figure 3. The results of two simulation runs in which both MTA and $B$ were fixed at their left ends and deformed by a vertical force acting at their free right end. Both MTs were 2 um long. MTA had the same Young's and shear moduli, whereas MT B corresponded to the realistic situation, where the shear is much lower than the Young's modulus. The forces applied on MT A and B were different, chosen so as to induce the same maximal deflexion on both MTs. The same force applied in both cases would produce $70 \%$ larger deformation of MT B than MT A. To compare the two shapes, the images of the two deformed MT were superimposed at both their ends. As can be seen, the shapes of the MTs under deformation are different. bending modulus, we explored the resistance of a single MT to random lattice defects. Such defects could occur during assembly, both in vitro and in vivo, and also under exposure of microtubules to UV light or oxidative stress during the photodynamic cancer therapy.

We randomly removed an increasing number of single tubulin units and followed the consequences on MT bending under a constant load. Links along and links between protofilaments were removed with the same probability. The MT was fixed on one of its ends and a force was applied at the other, free end. An MT missing $5 \%$ of its tubulin subunits is presented on Figure 4.

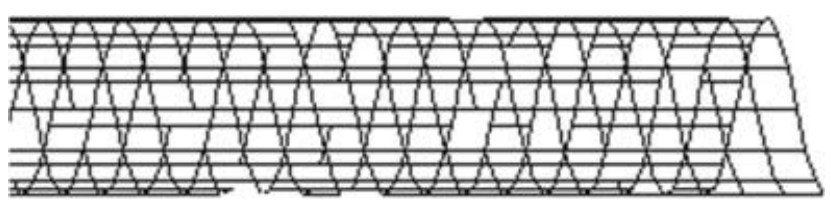

Figure 4. Close view of a MT missing $5 \%$ of randomly selected tubulin molecules.

Three different simulation runs were carried out for each percentage of missing elements. Since the choice of the elements that have to be suppressed is random, different numerical runs can give different results. Several such runs are necessary, since the mechanical properties of the MT highly depend on the location of the missing elements. For example, if only 13 elements were missing at the same distance from one end of the MT, the structure would break, independent of its length. This fact explains why increasing the percentage of missing elements increases the divergence between results of different numerical runs. Figure 5 depicts the dependence of the maximal deflection under a constant load as a function of the percentage of missing elements.

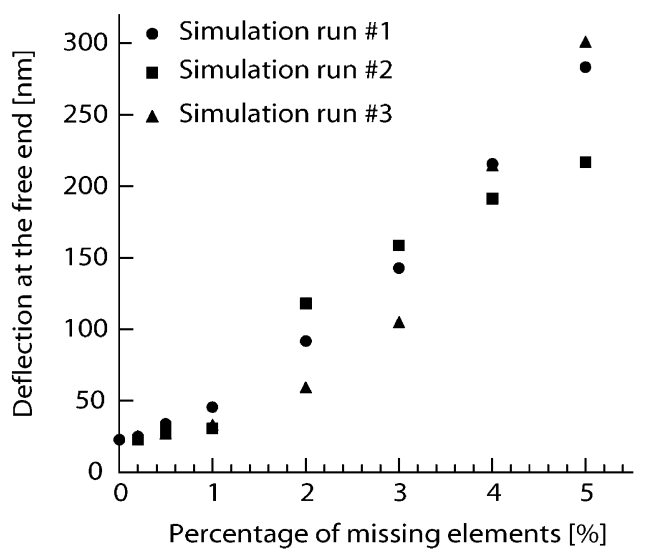

Figure 5. Influence of the number of missing elements in the deflection under a point load of $1 \mathrm{pN}$ at the free end of a $1 \mu \mathrm{m}$ long MT.

To explore the effect the number of protofilaments and the helicity plays in the mechanical properties, we modeled a 15:3 MT, with geometrical characteristics set according to Chretien et al. ${ }^{[6]}$ It is important to notice that in this form of MT, the protofilaments run helically, with a skew angle of $1.33^{\circ}$, around 
the principal axis of the MT, while in the most abundant, 13:3 MTs, they run parallel to the longitudinal axis. Our model treats $\alpha$ and $\beta$-tubulin molecules equally, so the presence of the seam in the microtubule structure is not taken into account, as it would require a precise knowledge of the difference in interactions between $\alpha-\alpha, \beta-\beta$ and $\alpha-\beta$ tubulin pairs.

For the simulation, the 15:3 MT had one of its ends blocked and a transverse force was applied at its free end. The values of the Young's and the shear modulus were set according to Mickey and Howard ${ }^{[14]}$ and Kis et al., ${ }^{[11]}$ respectively.

Simulations revealed that this type of MT deflects not only in the plane where the force is applied but also perpendicularly to it. This effect is depicted in Figure 6. Under the application of a

a)
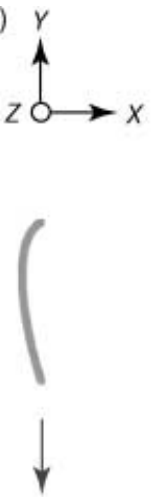

Figure 6. Application of a vertical load (arrow) at the end of a pinned MT induces deformations in two perpendicular planes.

load in the $y$ direction, the helical microtubule will deform in the direction of the load and also perpendicular to it, in the $x$ direction, as shown in Figure $6 \mathrm{a}$. Figure $6 \mathrm{~b}$ shows the MT in the $y z$ plane, with deformation in the direction of the applied force (Figure $6 \mathrm{~b}$ shows a side view of the deformed MT). Such a peculiar way of deformation under perpendicular stress makes these microtubules prone to buckling. As a consequence, this type of microtubules could be more sensitive to mechanical stimuli then the 13 protofilament ones, showing why they are associated with the sensitivity of $C$. elegans to touch. ${ }^{[4]}$

Herein, we have shown that FEM can successfully be applied to explore the behavior of highly complex structures such as microtubules at a microscopic scale. The crucial requirement is, of course, the knowledge of the objects geometry and the mechanical properties of the assembling units. The model was adjusted to correspond to recently published data on the mechanical properties of microtubules.

Our simulations have shown that the assumptions made by Kis et al. ${ }^{[11]}$ for analyzing the AFM results correspond well with the FEM simulations. In a second step, we applied this model to examine some situations which are difficult to explore experimentally, such as the modification of the shear modulus of the MT. FEM simulations permitted to predict the consequences of such a change on the bending of MT under a well defined mechanical stress. These simulations have shown that the assumption that has previously been made in considering MT as isotropic homogenous structures can lead to large errors in the interpretation of the experimental results.

The FEM simulation also permitted us to estimate the sensitivity of MT to the removal of an increasing number of the subunits which compose them. The simulation shows that MTs are relatively sensitive to lattice defects. The suppression of only $1.5 \%$ of the building units induces a $100 \%$ increase in bending under a tangential load.

Finally, the numerical exploration of an "exotic" MT type, the 15:3 MT, has demonstrated that the exposure of this MT to a tangential stress provokes bending in two different planes, one which is in the plane of the long axis of the MT and in another plane which is orthogonal to the first one. This phenomenon is due to the helical wrapping of the protofilaments along the MT principal axis. It also should exist for all the varieties of MT which present a skew angle different from zero. This hypothesis could be tested in future by performing measurements on MT assembled under different conditions in vitro. ${ }^{[20]}$ For example, using GMPCPP (guanylyl $\alpha, \beta$-methylenediphosphonate), a slowly hydrolyzable analogue of GTP (guanosine 5'-triphosphate) leads exclusively to the assembly of 14-protofilament MT.

These very encouraging preliminary results on MT, demonstrate that FEM can be a very convenient numerical method to explore the mechanical behavior of "large" biological structures.

\section{Computational Methods}

Microtubules without MAPs were simulated using the ASDL ANSYS $^{\circledR} 5.7$ programming language (http://www.ansys.com). Every single tubulin molecule was simulated by elements sharing the nominal dimensions and physical properties of tubulin. The dimensions of single tubulin molecules and their connecting angles were set according to Chretien and Wade. ${ }^{[6]}$ We used the BEAM4, which is a 3D uniaxial element with tension, compression, torsion, and bending capabilities, with every element corresponding to a single tubulin molecule. The material constants for each tubulin molecule were calibrated according to the value of $2 \mathrm{GPa}$ for the Young's modulus ${ }^{[15]}$ from measurements on long microtubules without MAPs, ${ }^{[14]}$ and 1.4 $\mathrm{MPa}$ for the shear modulus from the AFM measurements. ${ }^{[1]}$ Lateral contacts between the subunits are made between $\alpha-\alpha$ and $\beta-\beta$ tubulin molecules, except for a "seam" of $\alpha-\beta$ contacts. Interactions between isolated $\alpha-\alpha, \beta-\beta$ and $\alpha-\beta$ molecules are unknown at this time, mostly because of the inability to separate $\alpha$ and $\beta$-tubulin. Our model therefore doesn't make a distinction between $\alpha$ and $\beta$-tubulin.

The numerical runs where these quantities were set to different values are indicated in the corresponding result section. Boundary conditions for different simulations are also described above. To permit the simulation of large deformations, the nonlinear solver option was activated during the solution process. The ASDL model is available on request.

\section{Acknowledgements}

Milan Jirašek, Andrzej J. Kulik, J.P. Aimé and P. De Los Rios are gratefully acknowledged for highly constructive discussions, Ricar- 
do Perez for valuable input on ANSYS programming techniques, and $W$. Benoit for continuing support. This work has been financially supported by grant 2000-065160.01 and the "Nanoscale Science" NCCR of the Swiss National Science Foundation.

Keywords: finite element analysis - microtubules $\cdot$ molecular modeling $\cdot$ proteins $\cdot$ scanning probe microscopy

[1] B. A. Afzelius, P. L. Bellon, S. Lanzavecchia, J. Cell Sci. 1990, 95, 207-217.

[2] P. R. Burton, R. E. Hinkley, G. B. Pierson, J. Cell. Biol. 1975, 65, 227- 233.

[3] U. Eichenlaubritter, J. B. Tucker, Nature 1984, 307, 60-62.

[4] C. Savage, M. Hamelin, J. G. Culotti, A. Coulson, D. G. Albertson, M. Chalfie, Genes Dev. 1989, 3, 870-881.

[5] J. B. Tucker, M. J. Milner, D. A. Currie, J. W. Muir, D. A. Forrest, M. J. Spencer, Eur. J. Cell Biol. 1986, 41, 279-289.

[6] D. Chretien, R. H. Wade, Biol. Cell. 1991, 71, $161-174$

[7] E. M. Mandelkow, Current Opinion in Cell Biology 1995, 7, 72-81.

[8] M. Kurachi, M. Hoshi, H. Tashiro, Cell Motil. Cyt. 1995, 30, 221 - 228.

[9] P. Venier, A. C. Maggs, M.-F. Carlier, D. Pantaloni, J. Biol. Chem. 1994, 269, $13353-13360$.

[10] A. Vinckier, C. Dumortier, Y. Engelborghs, L. Hellemans, J. Vac. Sci. Tech. B 1996, 14, $1427-1431$

[11] A. Kis, S. Kasas, B. Babic, A.J. Kulik, W. Benoît, G. A.D. Briggs, C. Schönenberger, S. Catsicas, L. Forró, Phys. Rev. Lett. 2002, 89, 248101.

[12] J. Mizushima-Sugano, T. Maeda, T. Miki-Noumura, Biochimica et Biophysica Acta 1983, 755, 257-262.

[13] E. Nogales, M. Whittaker, R. Milligan, K. Downing, Cell 1999, 96, 79-88.

[14] B. Mickey, J. Howard, J. Cell. Biol. 1995, 130, 909-917.

[15] J. Howard, Mechanics of Motor Proteins and the Cytoskeleton, Sinauer Associates, Inc., Sunderland, MA, 2001.

[16] J. C. Kurz, R. C. Williams, Biochemistry 1995, 34, 13374-13380.

[17] H. Felgner, R. Frank, M. Schliwa, J. Cell Sci. 1996, 109, 509-516.

[18] R. Schultheiss, E. Mandelkow, J. Mol. Biol. 1983, 170, 471 - 496.

[19] Y. H. Song, E. Mandelkow, Proc. Natl. Acad. Sci. U.S.A. 1993, 90, $1671-1675$.

[20] P. Meurer-Grob, J. Kasparian, R. H. Wade, Biochemistry 2001, 40, 8000 8008

[21] J.M. Gere, S.P. Timoshenko, Mechanics of materials, PWS-Kent, Boston, 1990.

Received: April 16, 2003 [Z799]

Revised: October 23, 2003

\section{A Strategy for the Measurement of the Vibrations of a Muoniated Radical Centre: Experimental Evidence}

\author{
Upali A. Jayasooriya, ${ }^{*[a]}$ Francis L. Pratt, ${ }^{[b]}$ \\ Georgina M. Aston, ${ }^{[a]}$ Susan Hall, ${ }^{[a]}$ Penny L. Hubbard, ${ }^{[a]}$ \\ and Martin McCoustra ${ }^{[c]}$
}

The positive muon is a particle, chemically analogous to a proton, with a spin of one half and a mass of about one ninth that of the proton. It decays with a radioactive lifetime of about $2.2 \mu \mathrm{s}$, by emitting a positron (detected by an array of plastic scintillation detectors). The most probable direction of positron emission is the spin direction of the muon at the moment of decay. Therefore, with the use of spin-polarised muon beams, it is possible to monitor the evolution of the muon spin after implantation in a sample. ${ }^{[1,2]}$ Intense, highly spin-polarised muon beams are produced at sites such as the ISIS facility at the Rutherford Appleton Laboratory, and these allow the consequences of muon implantation to be studied. After implantation into a sample, the muon may exist without chemical change. In this case, it mimics a proton and may form adducts by muonation. ${ }^{[3]}$ Alternatively, the muon may pick up an electron to form muonium, $\left(\mu^{+} e^{-}\right)$, a light isotope of the hydrogen atom, which can add to the unsaturated sites of molecules to give radical species. ${ }^{[4,5]}$ The muon probes the properties of the resulting radical via its hyperfine interaction with the unpaired electron. Therefore, the identification of the radical species that is formed becomes of paramount importance. The technique for addition site determination in the case of chemical systems is dependent on ab initio calculations, which predict the magnitude of the site-dependent hyperfine interaction; this can then be compared with the experiment. This has been quite a successful technique, as far as the muoniated organic radicals are concerned.

However, our muonium implantation studies with various organometallic systems, including metallocenes such as ferrocene, have highlighted the need for an independent method of determination of the muon addition site. This is because the $a b$ initio methods are less reliable as prediction tools of muon/ muonium addition sites when molecules with heavy atoms are involved. Clearly, an independent method of site determination is called for, if the $\mu S R$ techniques are to be widely applied to inorganic and organometallic compounds. The experimental results presented herein show the possibility of development of just such a method of measurement.

[a] Dr. U. A. Jayasooriya, Dr. G. M. Aston, S. Hall, Dr. P. L. Hubbard School of Chemical Sciences, University of East Anglia, Norwich NR4 7TJ (UK) Fax: (+44)-1603-592003

E-mail:u.jayasooriya@uea.ac.uk

[b] Dr. F. L. Pratt

ISIS Muon Facility, Rutherford Appleton Laboratory, Chilton, Didcot, Oxon OX11 OQX (UK)

[c] Dr. M. McCoustra

School of Chemistry, University of Nottingham, University Park, Nottingham NG7 2RD (UK) 